\title{
The employment impact of the COVID-19 pandemic on Americans with MS: Preliminary analysis
}

\author{
Malachy Bishop* and Stuart P. Rumrill \\ Department of Rehabilitation Psychology and Special Education, University of Wisconsin-Madison, Madison, \\ WI, USA
}

Received 28 July 2020

Accepted 17 November 2020

\begin{abstract}
.
BACKGROUND: The Coronavirus disease 2019 (COVID-19) pandemic has been associated with unprecedented social and economic impacts in the United States, including significant cuts to the labor force, beginning in the spring of 2020.

OBJECTIVE: In this study we examined the employment effects of the pandemic on Americans with multiple sclerosis (MS), a population that has historically experienced low rates of employment relative to the general population.

METHODS: Based on a survey of a convenience sample of 69 members of the National Multiple Sclerosis Society, we examined employment, health, and economic experiences between May and August, 2020.

RESULTS: The results suggest potentially significant job loss. Specifically, in the present sample approximately $20 \%$ of participants who were previously employed reported losing employment as a result of the pandemic, and approximately $40 \%$ reported other work changes. At elevated risk for job loss were those with lower levels of education, African American workers, and workers reporting Hispanic, Latinx, or Spanish origin. Almost two-thirds of the participants reported that the pandemic had affected their financial status and over half (53.6\%) reported that the pandemic had affected their ability to access their MS healthcare.

CONCLUSION: We discuss the implications and potentially lasting effects of the current, complex employment situation faced by Americans living with MS, and the broader considerations for vocational rehabilitation professionals.
\end{abstract}

Keywords: Multiple sclerosis, COVID-19 pandemic, employment, healthcare

\section{Introduction}

The Coronavirus disease 2019 (COVID-19) pandemic has been associated with unprecedented changes to daily life in the United States and globally. These changes have resulted in significant and, at the time of this writing, ongoing social and economic impacts. Among the consequences of COVID-19 in the US were rapid and deep cuts to the US labor

\footnotetext{
*Address for correspondence: Malachy Bishop, Ph.D., CRC, University of Wisconsin-Madison, Department of Rehabilitation Psychology and Special Education, 1000 Bascom Mall, Room 409, Madison, WI 53706, USA. E-mail: mlbishop4@wisc.edu.
}

force in March and April of 2020. The impact of the COVID-19 pandemic on the U.S. labor market has been profound, affecting all employment sectors, and particularly affecting populations characterized by the intersections of race, poverty, and disability (Filanoski, 2020a). The full extent, duration, and longer-term economic and social impacts of the COVID-19 pandemic are unpredictable and depend upon rapidly shifting circumstances.

Rehabilitation counseling professionals providing vocational services in the present environment are faced with exceptionally challenging circumstances in a restricted and fluid vocational landscape. Effective vocational rehabilitation counseling during the 
COVID-19 pandemic requires broad and current understanding of national and local labor markets, attention to the impacts on the employment of persons with specific disabilities and chronic illnesses and the specific labor sectors affected, and heightened awareness of the increased health risks associated with employment, particularly for those with chronic conditions.

Unfortunately, national employment data on persons with disabilities generally provide broad and time-delayed information that does not differentiate by functional or diagnostic categories. For example, as with most national data sets, in the U.S. Bureau of Labor Statistics Household Data on Employment Status and Disability Status (U.S. Bureau of Labor Statistics [USBLS], September 23, 2020), disability is defined using an aggregate definition based on having at least one of several conditions or limitations in various tasks. In the present, fluid national labor situation, such national-level and aggregate data are important and helpful, but they are unable to provide information about the experiences of people with specific conditions or disabilities.

In the spring of 2020, as the US entered a widespread period of restricted activity and voluntary or enforced quarantine and the initial labor impact of the COVID-19 pandemic was developing, we were interested in exploring and understanding the employment and psychosocial experiences of the pandemic among Americans with multiple sclerosis (MS). We initiated a national survey to explore these experiences. In this article we present employment data based on this survey. The purpose of this article is to provide a snapshot, albeit a limited one due to the size of the sample, of the effects of COVID-19 on the employment situation of a sample of Americans with MS.

Americans living with MS have historically experienced low employment participation rates relative to the general population (Rumrill \& Bishop, 2019). Understanding the employment effects of COVID-19 on this already at-risk population is important in terms of developing and delivering effective rehabilitation counseling interventions with this population. The results also have broader applicability for working with people with other chronic health conditions.

\subsection{Multiple sclerosis}

Multiple sclerosis is a chronic, inflammatory, demyelinating, and neurodegenerative autoimmune disease of the central nervous system (CNS). MS is characterized by intermittent and recurrent episodes of inflammation that result in the destruction of CNS myelin and the underlying CNS axons (Lanssmann, 2011; Lee \& Dunn, 2013). The disease is associated with a wide range of symptoms and is frequently accompanied by progressively increasing functional limitations affecting multiple functions and systems (Murray, 2016; Rumrill \& Bishop, 2019). MS is among the most prevalent neurological disorders, affecting an estimated 2.3 million people worldwide (Murray, 2016) and over 900,000 Americans (National Multiple Sclerosis Society [NMSS], 2018). Indeed, MS is the most common non-traumatic neurological disease of young adults in the world. Approximately 10,000 new cases of MS are diagnosed annually in the US (Kalb, 2016). Although MS may be diagnosed at any age, initial symptoms are most often evident during early adulthood, typically between the ages of 20 and 50 (Kalb, 2016; Schapiro, 2003). Therefore, the disease typically affects working aged, early- to mid-career adults. Most frequently diagnosed in the US among those of European origin, MS is seen in most ethnic groups including African Americans, Asians, and those of Hispanic or Latinx ethnicity (DeLuca \& Nocentini, 2011; Multiple Sclerosis Coalition, 2015).

\subsection{Employment and MS}

As is the case for persons with disabilities and chronic illnesses in the US generally (Strauser, 2014), Americans with MS face disparate opportunities for, and a range of barriers to, participation in the labor force, even during periods of economic growth or stability (Rumrill \& Bishop, 2019; Rumrill et al., 2005). More than $90 \%$ of individuals with MS in the United States have employment histories, and approximately $60 \%$ were working at the time of diagnosis (Rumrill et al., 2008), however, 50-80\% of people with MS who are working at the time of diagnosis are no longer in the workforce within 10 years, and approximately $40 \%$ leave employment in the first three years after diagnosis (McFadden et al., 2012; Strober, 2020). Over the past decade, the employment rate among US adults with MS has remained at approximately 40\% (Bishop et al., 2016; Strober, 2020).

Employment is an important contributor to physical and psychosocial health and quality of life, providing access to economic security, social participation, access to health insurance and health care, and contributing to quality of life (QOL; Bishop, et al., 2019). Indeed, in a recent large national survey of National MS Society members, employment 
was identified among the domains most important to QOL (Bishop et al., 2019). The fact that adults with MS consistently experience significantly higher levels of unemployment and underemployment than the general population can have significant psychosocial, health, and economic consequences.

The consequences of early career exit are particularly noteworthy in the face of research that shows that, among unemployed people with MS, 75\% left their jobs voluntarily, often before their symptoms made them incapable of working, $80 \%$ reported that they retain the ability to work, and up to $75 \%$ reported that they would like to return to work (Roessler et al., 2002; 2008). Premature career discontinuation has been associated with significant negative and long-term effects on financial stability, mental and physical health, community access and participation, and QOL (Bishop et al., 2015). Because the typical age of MS onset occurs early- to mid-career, the total economic impact for the individual with MS is greater than for those with other, later-onset chronic neurological diseases. Kobelt et al. (2006) estimated that the lost productivity costs of MS due to early retirement approximated $\$ 15,000$ per patient per year, and that, together with sick leave and reduced work time (10\%), early retirement and employment loss accounts for approximately $44 \%$ of the cost of MS

There has been extensive research, particularly over the past two decades, aimed at identifying the barriers to work faced by people with MS, the health, economic, and QOL benefits of working, the reasons for and consequences of leaving work, and the resources and interventions that help people with MS to stay employed (Bishop et al., 2016; Rumrill et al., 2008; Rumrill et al., 2013; Rumrill \& Bishop, 2019; Strober, 2020). However, the current COVID-19 situation introduces unique elements and barriers to employment for people living with MS. The pandemic has also heightened awareness and concerns about, and complicated, at least temporarily, the relationship between work and health. For example, although employment has generally been associated with better health among persons with MS (e.g., Dorstyn et al., 2019), continued employment during the time of the COVID-19 pandemic may be associated with an increased risk of health consequences. According to Moss et al. (2020), people living with MS may be at higher risk for health consequences from COVID-19 than the general public due to existing neurologic disability, the impact of disease modifying therapies on COVID-19 susceptibility and severity, and the effects of COVID-19 on ongoing MS disease activity. Furthermore, the capacity to maintain the good health and function necessary to remain employed is threatened for many people living with MS. As is often the case for persons with chronic illnesses, many people with MS require access to regular (and relatively frequent) medical services, including infusions, physical therapy, occupational therapy, and homecare services to maintain their health. Access to these supports may be disrupted as a result of the pandemic (Moss et al., 2020), thus potentially decreasing baseline health.

In order to evaluate the employment impact of the COVID-19 pandemic on people living with MS, and to begin to explore the interacting effects of health, employment, and healthcare access, in the present study we surveyed a sample of Americans with MS about their employment, health, and economic experiences between May and August, 2020. The data represent the first wave in what we anticipate will be a larger, longitudinal analysis of these impacts over the next two years. In the following sections, we present the methods and results of this initial survey. We then discuss the implications of the current, complex employment situation faced by Americans living with MS, and the broader considerations for vocational rehabilitation professionals. We address the following research questions:

1. How has the COVID-19 pandemic, and related social and labor market responses, affected the employment and economic status of Americans with MS?

2. Has the COVID-19 pandemic affected healthcare and treatment access in a manner that may affect engagement in employment?

\section{Method}

\subsection{Procedures}

The Institutional Review Board (IRB) of the University of Wisconsin-Madison reviewed and approved this longitudinal study. Participants in the present analysis were recruited between May 15 and August 2, 2020 through the National Multiple Sclerosis Society's research portal. The Society provides opportunities for members to participate in research through a website that details ongoing, approved research studies. Members interested in participating in the present study, based on a brief description of the research posted on the website, were asked to select the link to the survey questionnaire, which was 
hosted using the Qualtrics Survey Hosting Service (2020; Qualtrics, Provo, UT). Participants were asked to read an online consent form and indicate consent before proceeding to the survey questionnaire. The following inclusion criteria were applied: (a) age of 18 or older, (b) having a diagnosis of multiple sclerosis. Participants were offered a $\$ 10.00$ electronic gift card for their participation.

\subsection{Participants}

We present the results for 69 people who met the inclusion criteria and responded to the online survey. Participants' ages ranged from 22 to 77 and the mean age was $43.43(S D=12.71)$. The majority of participants were female (75.4\%) and reported their ethnicity as White (81.2\%). Over ten percent of the participants, respectively, reported their race/ ethnicity as African American (11.6\%) or reported being of Hispanic, Latinx, or Spanish origin (11.9\%). Most participants reported their marital status as married $(56.5 \%) ; 2.9 \%$ reported living with a partner and the remainder identified themselves as single/never married (27.5\%), divorced (8.7\%), separated (1.4\%), or widowed (2.9\%). Participants represented 24 U.S. states and primarily resided in suburban $(52.9 \%)$ or urban $(38.2 \%)$ areas, with $8.8 \%$ describing their residential area as rural. Participants reported their highest level of education as follows: $1.4 \%$ less than high school, $8.7 \%$ high school graduate, $18.8 \%$ some college but no degree, $13.0 \%$ Associate's degree, $30.4 \%$ Bachelor's degree, and $27.5 \%$ graduate or professional degree.

We asked participants to describe their pre-pandemic employment status as follows: "Which one of the following best describes your employment status? (Note: We realize that, for many people, the employment situation has changed recently due to COVID-19. Tell us here about your usual employment status (prior to any COVID-19-related changes.))" Participants' 'usual' employment status was reported as follows: employed or self-employed fulltime (33.3\%); employed or self-employed part-time $(20.3 \%)$; unpaid or volunteer work $(1.4 \%)$; unemployed, and looking for work $(10.1 \%)$; unemployed, and not looking for work (5.8\%); unemployed and receiving disability benefits $(11.6 \%)$; homemaker (8.7\%); full-time student (2.9\%); retired, not disabled (4.3\%), and other (1.4\%). Among those who reported being employed either full- or part-time, the mean number of years employed in their current position was $6.14(S D=5.43)$.
Annual total household income, before taxes, was reported as follows: $17.6 \%$ reported less than $\$ 25,000 ; 30.9 \%$ reported $\$ 25,000$ to $\$ 49,999 ; 29.4 \%$ reported $\$ 50,00$ to $\$ 74,999 ; 10.3 \%$ reported $\$ 75,000$ to $\$ 99,999$; and $11.8 \%$ reported above $\$ 100,00$. Most participants $(62.3 \%)$ reported having private health insurance; $24.6 \%$ had Medicaid, $14.5 \%$ had Medicare, and one participant had health insurance through the Department of Veterans Affairs or TRICARE. With respect to benefits programs, $14.5 \%$ were receiving Supplemental Security Income (SSI), 27.5\% Social Security Disability Insurance (SSDI), 7.2\% Workers Compensation benefits, and $10.1 \%$ retirement benefits/pension.

The mean age at diagnosis with MS was 34.7 years $(S D=13.31)$. Over two-thirds $(68.1 \%)$ reported the course of their MS as relapsing-remitting, and $26.1 \%$ as progressive, including primary $(14.5 \%)$ and secondary $(11.6 \%)$ progressive, and the remainder reported being unsure. The majority $(88.1 \%)$ reported that they were currently using disease-modifying medications. Over one-third reported having been diagnosed with co-occurring depression (36.2\%), and $37.7 \%$ with an anxiety disorder.

\subsection{Instrument and data analysis}

The present analysis involved data that were collected as part of a broader study that evaluated additional psychosocial information beyond the focus of the present article. The survey questionnaire included 54 items, and it had several sections addressing participant's demographic characteristics, healthcare and employment-related experience variables, course of MS and treatment, QOL and psychosocial status, and several brief standardized measures. For the purpose of this article, we focus on the demographic, healthcare, and employmentrelated variables. The data were analyzed using IBM SPSS Statistics (Version 26). Descriptive statistics and non-parametric chi square analyses were computed to provide characteristics of the sample and address the research questions.

\section{Results}

The first research question addressed the degree to which the COVID-19 Pandemic and related social and labor market responses affected the employment and financial status of Americans with MS. Almost one-half of the sample $(47.8 \%)$ reported that 
the COVID-19 pandemic resulted in some change in their employment situation. Excluding the participants who reported their usual employment situation as retired, unemployed and receiving disability benefits, and those in active military service, 57.9\% reported some employment-related change. Specifically, 29.8\% reported being no longer employed as a result of the pandemic. Among the participants who reported that they were employed full-time prior to COVID-19, 17.4\% reported that they were no longer employed as a result of the COVID-19 pandemic, and $39.1 \%$ reported other employment changes. Among the participants reporting part-time employment, $21.4 \%$ reported having lost their positions, and $42.9 \%$ reported experiencing another change in employment. When participants were asked to describe the employment-related changes, the most frequently reported changes included working less hours or fewer shifts, followed by shifting to workfrom-home, and changing departments or positions with their employer.

Examination of demographic variables and job loss due to the COVID-19 pandemic revealed that, among those who reported their usual employment status (prior to the COVID-19 pandemic) as employed fullor part-time, there were no significant differences in terms of gender. Participants who reported their ethnicity as African Americans reported a higher rate of job loss (25.0\%) compared to those reporting their ethnicity as White (19.4\%), but the difference was not statistically significant. Those reporting being of Hispanic, Latinx, or Spanish origin, however, did report a statistically significantly higher rate of job loss $(60 \%)$ relative to the remainder of the participants $\left(18.8 \% ;\left(\chi^{2}(1, N=37)=3.997, p=.046\right)\right.$. Education level was also associated with job loss. Specifically, among those working full- or part-time prior to the pandemic, whereas $14.3 \%$ of workers who had completed a college degree (Associates, bachelor's, or professional degree) reported having lost their job as a result of COVID-19, the percentage among those with no degree was significantly higher, at $55.6 \%$ $\left(\chi^{2}(1, N=37)=6.302, p=.012\right)$.

Almost two-thirds of the participants $(65.2 \%)$ reported that the COVID-19 pandemic had affected their financial status. An additional $17.4 \%$ reported that their insurance status had been affected. In terms of current financial status, while 52.2\% reported that they were "able to meet expenses", $40.6 \%$ reported "some difficulty meeting expenses", and $7.2 \%$ were "unable to meet expenses". Among those who reported their typical employment situation as employed part- or full-time but who had lost their job due to COVID-19, $40.9 \%$ reported some difficulty meeting expenses, and $9.1 \%$ reported being unable to meet expenses.

In the second research question, we evaluated the extent to which health and treatment access barriers have affected the participation of Americans with MS in the labor market. Over half of the participants (53.6\%) reported that the COVID-19 pandemic had affected their ability to access their MS healthcare, and $37.7 \%$ reported that their ability to access MS treatments or medications had been affected. Several participants commented on their barriers with respect to accessing healthcare, and these comments suggested that the impacts included appointment cancellations and delays or rescheduling, visits being held via on-line portals rather than in-person, and fear of traveling to meet with providers during the pandemic.

\section{Discussion}

The convenience nature of the sampling procedure and the complete reliance on self-report data are noteworthy limitations of the present study. Also, the small respondent sample that was drawn from the membership organization National MS Society may not be representative of the broader population of adult Americans with MS. However, the results of this analysis suggest several areas of potential concern. First and almost certainly foremost, approximately $20 \%$ of employed participants in this study reported losing employment as a result of the pandemic. Although the other employment changes reported by approximately $40 \%$ of employed participants were not consistently associated with reduced work (e.g., working from home), many reported working less hours or fewer shifts. The present data suggest a significant loss of employment among a population that has historically experienced relatively lower rates of employment when compared with non-disabled people (Strober, 2020). At elevated risk for job loss in the present sample were those with lower levels of education, African American workers, and workers reporting Hispanic, Latinx, or Spanish origin. Finally, it is particularly troubling that almost two-thirds of the sample reported that the COVID-19 pandemic had affected their financial status, with half of those participants who had been employed but who had lost their job due to COVID-19 reporting some difficulty meeting expenses, and almost 1 -in-10 reporting being 
unable to meet expenses. Along with the impacts on insurance status, the present results suggest that a relatively large proportion of persons living with MS may be facing significant health and financial vulnerability as a result of the pandemic.

A potential longer-term consequence, in terms of work re-entry for persons with MS who have lost their employment, is the potential for a continued labor market contraction to increase the long-term risk of permanent unemployment for many Americans with MS. This risk is highlighted in the context of recently released US labor data (USBLS, September 23,2020 ), which suggests that, in addition to the fact that the unemployment rate among working aged (aged 16-64) persons with a disability $(13.4 \%)$ is almost double that of those with no disability $(7.5 \%)$, the employment-to-population ratio, which reflects the percentage of people who are working relative to the total population, had decreased from August to September, while for working-age people without disabilities this ratio had increased slightly (Filanoski, 2020b). According to Houtenville (in Finaloski, 2020b) "the labor force participation rate is a bell-weather of the long-term employment prospects of people with disabilities," and the recent decline in this indicator suggests that a growing number of people with disabilities are no longer on furlough or actively looking for work, but are exiting the labor market. Houtenville predicted that "as the pandemic continues and more workers are permanently let go, we are likely to see further declines in the labor force participation rate.” In short, it appears that a significant number of Americans with MS may be experiencing a loss of employment at a time when rejoining the labor market is likely to remain more difficult than usual for the foreseeable future. This situation has potentially significant long-term effects on the economic, physical, and mental health of Americans affected by MS. Possibly further complicating the employment impact of COVID-19 are the impacts of restricted access to MS healthcare, inability to access MS treatments or medications, and the restrictions resulting from recommendations to limit social interactions on the part of those with chronic illnesses (especially those conditions like MS that stem from compromised immunologic functioning). Vocational rehabilitation counselors working with people with MS must be able to deliver direct-service interventions using distance and online technology, consider on-the-job accommodations and other employment modifications within the growing work-at-home context that has been necessitated by the pandemic, ensure that consumers with MS are accessing needed healthcare through telehealth delivery platforms if necessary, and understand the heightened risks for job loss and other employment changes that attend to workers with MS who have lower levels of education and/or are people of color.

Future longitudinal research is needed to track the effects that COVID-19 will have on the health and employability of people with MS over time. Changes in the US economy that cannot be predicted at the time of this writing will continue to shape the types and delivery platforms of vocational rehabilitation interventions that people with MS need in the post-COVID recovery. The intersectionality of socioeconomic status, race-ethnicity, access to healthcare, overall health and well-being, self-management, and labor force participation, as important as it has been prior to COVID-19, will take on greater importance as people with MS, the professionals who serve them, and the entire global community progress through the end stages and aftermath of our present, unprecedented worldwide health crisis.

\section{Acknowledgment}

The authors wish to thank the National Multiple Sclerosis Society and the individuals with multiple sclerosis who participated in this study for their assistance with this research.

\section{Conflict of interest}

The authors have no conflicts of interest to disclose.

\section{Funding}

Support for this research was provided by the Wisconsin Center for Education Research, University of Wisconsin-Madison. The contents of this article were developed in part through a grant from the National Multiple Sclerosis Society, New York, NY. The authors wish to thank the National Multiple Sclerosis Society and the individuals with multiple sclerosis who participated in this study.

\section{References}

Bishop, M., Fraser, R., Li, J., Rumrill, P. D., Burns, M., LaRocca, N., Frain, M., McDaniels, B., \& Lee, B. (2019). 
Life domains that are important to quality of life for people with multiple sclerosis: A population-based qualitative analysis. Journal of Vocational Rehabilitation, 51(1), 67-76. https://doi.org/10.3233/JVR-191026

Bishop, M., Rumrill, P. D., \& Timblin, R. I. (2016). Medical, psychosocial, and vocational aspects of multiple sclerosis: Implications for rehabilitation professionals. Journal of Rehabilitation, 82(2), 6-13.

Bishop, M., Rumrill, P. D., \& Roessler, R. T. (2015). Quality of life among people with multiple sclerosis: Replication of a three-factor prediction model. Work: Journal of Prevention, Treatment and Rehabilitation, 52, 757-765. https:// doi.org/10.3233/WOR-152203

DeLuca, J., \& Nocentini, U. (2011). Neurological, medical, and rehabilitative management in persons with multiple sclerosis. NeuroRehabilitation, 29, 197-219. https://doi. org/10.3233/NRE-2011-0695

Dorstyn, D. S., Roberts, R. M., Murphy, G., \& Haub, R. (2019). Employment and multiple sclerosis: A meta-analytic review of psychological correlates. Journal of Health Psychology, 24(1), 38-51. https://doi.org/10.1177/1359105317691587

Filanoski, K. (August 18, 2020a). nTIDE July 2020 Jobs Report: July numbers raise red flag for Americans with disabilities. Employment Policy and Measurement Rehabilitation Research and Training Center, Kessler Foundation and University of New Hampshire. https://researchondisability.org/epmrrtc/epm-news/2020/08/18/ntide-july-2020-jobs-report-julynumbers-raise-red-flag-for-americans-with-disabilities

Filanoski, K. (October 2, 2020b). nTIDE September 2020 Jobs Report: Unease rises as numbers fall for Americans with disabilities. Employment Policy and Measurement Rehabilitation Research and Training Center, Kessler Foundation and University of New Hampshire. https://researchondisabil ity.org/epm-rrtc/epm-news/2020/10/02/ntide-september2020-jobs-report-unease-rises-as-numbers-fall-foramericans-with-disabilities

Kalb, R. (2016). Living with multiple sclerosis: The psychosocial challenges for patients and their families. In B. Giesser (Ed.), Primer on multiple sclerosis (2nd ed., pp. 483-498). Oxford University Press.

Kobelt, G., Berg, J., Atherly, D., \& Hadjmichael, O. (2006). Costs and quality of life in multiple sclerosis: A cross sectional study in the United States. BMC Health Services Research, 3, 17.

Lanssmann H. (2011). Pathophysiology of inflammation and tissue injury in multiple sclerosis: What are the targets for therapy? Journal of the Neurological Sciences, 306(1-2), 167-169. https://doi.org/10.1016/j.jns.2010.07.023

Lee J. M., \& Dunn J. (2013). Mobility concerns in multiple sclerosis: Studies and surveys on US patient populations of relevance to nurses. US Neurology, 9(1), 17-23. https://doi. org/10.17925/USN.2013.09.01.17

McFadden, E., Horton, M. C., Ford, H. L., Gilworth, G., McFadden, M., \& Tennant, A. (2012). Screening for the risk of job loss in multiple sclerosis (MS): Development of an MS-specific
Work Instability Scale (MS-WIS). Multiple Sclerosis Journal, 18(6), 862-870. https://doi.org/10.1177/1352458511428463

Moss, B. P., Mahajan, K. R., Bermel, R. A., Hellisz, K., Hua, L. H., Hudec, T., Husak, S., McGinley, M. P., Ontaneda, D., Wang, Z., Weber, M., Tagliani, P., Cárdenas-Robledo, S., Zabalza, A., Arrambide, G., Carbonell-Mirabent, P., Rodríguez-Barranco, M., Sastre-Garriga, J., Tintore, M., ... Fitzgerald, K. C. (2020). Multiple sclerosis management during the COVID19 pandemic. Multiple Sclerosis Journal, 26(10), 1163-1171. https://doi.org/10.1177/1352458520948231

Multiple Sclerosis Coalition. (2015). The use of disease-modifying therapies in multiple sclerosis-principles and current evidence: A consensus paper. http://www.nationalmssociety.org/get media/5ca284d3-fc7c-4ba5-b005-ab537d495c3c/DMT_

Consensus_MS_Coalition_color

Murray, T. J. (2016). The history of multiple sclerosis: From the age of description to the age of therapy. In B. Giesser (Ed.), Primer on multiple sclerosis (2nd ed., pp. 3-10). Oxford University Press.

National Multiple Sclerosis Society (2018). MS prevalence. National Multiple Sclerosis Society. Retrieved May 23, 2018 from http://www.nationalmssociety.org/About-theSociety/MS-Prevalence.

Roessler, R. T., Rumrill, P. D., \& Hennessey, M. L. (2002). Employment concerns of people with multiple sclerosis: Building a national employment agenda (Report to the National Multiple Sclerosis Society, New York, NY): University of Arkansas and Kent State University, Center for Disability Studies.

Rumrill, P., \& Bishop, M. (2019). Multiple sclerosis: A highincidence immune-mediated disease of the central nervous system [Special Issue]. Journal of Vocational Rehabilitation, 51(1), 1-9. https://doi.org/10.3233/JVR-191020

Rumrill, P., Fraser, R., \& Johnson, K. (2013). Employment and workplace accommodation outcomes among participants in a vocational consultation service for people with multiple sclerosis. Journal of Vocational Rehabilitation, 39, 85-90. https://doi.org/10.3233/JVR-130646

Rumrill, P., Hennessey, M., \& Nissen, S. (2008). Employment issues and multiple sclerosis (2nd ed.). Demos.

Rumrill, P. D., Roessler, R., McMahon, B., \& Fitzgerald, S. (2005). Multiple sclerosis and workplace discrimination: The national EEOC ADA research project. Journal of Vocational Rehabilitation, 23, 179-187.

Schapiro, R. T. (2003). Managing the Symptoms of Multiple Sclerosis (4th ed.). Demos Medical Publishing.

Strober D. L. (2020). Determinants of unemployment in multiple sclerosis (MS): The role of disease, person-specific factors, and engagement in positive health-related behaviors. Multiple Sclerosis and Related Disorders, 46. Advance online publication. https://doi.org/10.1016/j.msard.2020.102487.

U. S. Bureau of Labor Statistics. (September 23, 2020). Household Data on Employment Status and Disability Status. https://www.bls.gov/news.release/empsit.t06.htm 